\title{
A Highly Active and Tolerable Neoadjuvant Regimen Combining Paclitaxel, Carboplatin, 5-FU, and Radiation Therapy in Patients with Stage II and III Esophageal Cancer
}

\author{
L. van de Schoot, MD, ${ }^{1}$ E. A. P. M. Romme, MD, ${ }^{1}$ M. J. van der Sangen, MD, ${ }^{2}$ \\ G. J. Creemers, MD, $\mathrm{PhD},{ }^{3}$ G. van Lijnschoten, MD, PhD, ${ }^{4}$ \\ O. J. Repelaer van Driel, MD, $\mathrm{PhD},{ }^{5}$ H. J. T. Rutten, MD, PhD, ${ }^{1}$ \\ and G. A. P. Nieuwenhuijzen, $\mathrm{MD}, \mathrm{PhD}^{1}$
}

\begin{abstract}
${ }^{1}$ Department of Surgery, Catharina Hospital, Eindhoven, The Netherlands ${ }^{2}$ Department of Radiotherapy, Catharina Hospital, Eindhoven, The Netherlands ${ }^{3}$ Department of Medical Oncology, Catharina Hospital, Eindhoven, The Netherlands ${ }^{4}$ Department of Pathology, Catharina Hospital, Eindhoven, The Netherlands ${ }^{5}$ Department of Surgery, Maxima Medisch Centrum, Eindhoven, The Netherlands
\end{abstract}

Background: The present phase II study aimed to assess the feasibility and efficacy of a new paclitaxel-based neoadjuvant chemoradiation regimen followed by surgery in patients with stage II-III esophageal cancer.

Methods: From January 2002 to November 2004, 50 patients with a potentially resectable stage II-III esophageal cancer received chemotherapy with paclitaxel, carboplatin, and 5-FU in combination with radiotherapy 45 Gy in 25 fractions. Surgery followed 6-8 weeks after completion of neoadjuvant treatment.

Results: Patient characteristics: male/female: 44/6, median age 60 years (34-75), median WHO $1(0-2)$, adenocarcinoma $(n=42)$, squamous cell carcinoma $(n=8)$. Toxicity was mild, and $84 \%$ of the patients completed the whole regimen. Forty-seven patients underwent surgery with a curative intention (transhiatal $n=44$, transthoracic $n=3$ ).

Pathologic complete tumor regression was achieved in 18 of 47 operated patients $(38 \%)$. R0 resection was achieved in 45 of 47 operated patients $(96 \%)$. There were four postoperative deaths (8.5). Postoperative complications were comparable with other studies. After a median follow-up of 41.5 months (21-59) estimated 3- and 5-year survival on an intention-to-treat basis was 56 and $48 \%$. Estimated 3-year survival in responders was $61 \%$, in nonresponders $33 \%$.

Conclusion: This novel neoadjuvant chemoradiation regimen for treatment of patients with stage II-III esophageal cancer is feasible. Results are encouraging with a high pathologic complete tumor regression and $\mathrm{R} 0$ resection rate and an acceptable morbidity and mortality. Preliminary survival data are very promising.

Key Words: Esophageal cancer-Neoadjuvant chemoradiation-Paclitaxel-Pathologic complete response-Tumor regression grade.

Received March 16, 2007; accepted July 27, 2007; published online: September 26, 2007.

On behalf of the hospitals affiliated to the Comprehensive Cancer Center South, The Netherlands

Address correspondence and reprint requests to: L. van de Schoot, MD; E-mail: scho4190@planet.nl

Published by Springer Science+Business Media, LLC $\odot 2007$ The Society of Surgical Oncology, Inc.
The prognosis of patients with esophageal cancer who undergo surgery with a curative intention is poor, with a 5-year survival rate of $5-20 \% .^{1}$ In recent decades, several efforts have been made to improve outcome. Improved and standardized surgical techniques and supportive care as well as concentration of 
care have contributed to a better outcome; however, further improvement of survival from a single modality approach seems unlikely. ${ }^{2,3}$

Neoadjuvant chemoradiation has become the focus of interest to improve long-term survival and reduce recurrence rates. Despite a large number of phase II and several phase III trials, the role of neoadjuvant chemoradiation remains unclear. Until now, only one phase III trial demonstrated a significant survival advantage. ${ }^{4}$ All neoadjuvant regimens used in these phase III trials were cisplatin-based, which leads to frequent toxicity-related dose reductions and delays and relatively low pathologic complete response rates (10-28\%).

New neoadjuvant chemoradiation regimens including drugs such as paclitaxel have shown encouraging complete response rates (29-39\%) and R0 resection rates. ${ }^{5-7}$ Since pathologic complete response and $\mathrm{R} 0$ resection have been shown to be associated with improved survival, ${ }^{8,9}$ it can be expected that these new regimens lead to better survival rates. Therefore, the optimal neoadjuvant treatment regimen with high $\mathrm{pCR}$ rates and minimal toxicity has to be determined.

Paclitaxel interferes with mitotic spindle function by enhancing the rate and yield of microtubule assembly and preventing microtubule depolymerization. ${ }^{10}$ Paclitaxel is a potent radiosensitizer because it synchronizes cells at $\mathrm{G} 2 / \mathrm{M}$, the phase of the cell cycle that is particularly sensitive to radiotherapy. ${ }^{11,12}$ Furthermore, paclitaxel radiosensitization persists well after the period of G2/M synchronization, suggesting that other factors may also relate to its ability to enhance radiotherapy. ${ }^{13}$ The p53 gene is required for initiation of apoptosis in response to most chemotherapeutic agents and radiotherapy. In vitro, paclitaxel-mediated blockade at the G2/M has shown to activate cell-cycle control pathways that induce apoptosis independent of p53. ${ }^{14}$

Therefore, paclitaxel-based neoadjuvant chemoradiation seems a rational treatment approach for esophageal cancer. A recent study by Meluch et $\mathrm{al}^{6}{ }^{6}$ has reported an encouraging complete response rate of $38 \%$ with a paclitaxel-based regimen. However, toxicity was severe as only $54 \%$ of the patients received a full dose of the neoadjuvant regimen, because of complicating esophagitis and leucopenia. The goal of this study was to assess the feasibility and efficacy of a new paclitaxel-based neoadjuvant chemoradiation protocol in which we applied a reduced chemotherapy dose compared with the protocol of Meluch et al. ${ }^{6}$

\section{PATIENTS AND METHODS}

\section{Patients}

Referral to our institute was accomplished as a result of agreements between hospitals affiliated to the Comprehensive Cancer Centre South in the Netherlands to concentrate surgical care of patients with esophageal cancer. The protocol of this prospective clinical phase II trial was approved by the Medical Ethics Committee, and from all patients written informed consent was obtained. All consecutive patients with a potentially resectable stage II and III esophageal cancer who were referred to our Centre between January 2002 and November 2004 were found eligible.

Further eligibility criteria included: patient age between 18 and 75 years, WHO performance status $\leq 2$, written informed consent, central venous catheter, and adequate hematological, renal, and hepatic functions defined as white blood cell count > $3.5 \times 10^{9} / \mathrm{L}$, platelet count $>100 \times 10^{9} / \mathrm{L}$, creatinine level $<120 \mu \mathrm{mol} / \mathrm{L}$ and/or creatinine clearance $>60$ $\mathrm{mL} / \mathrm{min}$, and normal hepatic enzyme levels. Patients with distant metastatic disease or tumor positive supraclavicular lymph nodes were excluded from entry into the protocol. However, M1a disease in the regional lymph nodes was not.

Pretreatment staging evaluation included clinical examination, upper gastrointestinal endoscopy with histological biopsies of the tumor, computed tomography (CT) scan of chest and upper abdomen, and ultrasound of the neck. Endoscopic ultrasound (EUS) was performed when a T1 tumor could not be excluded by conventional techniques. More recently, PET imaging was introduced to exclude distant metastases.

\section{Neoadjuvant Treatment}

The neoadjuvant regimen consisted of paclitaxel $175 \mathrm{mg} / \mathrm{m}^{2}$ intravenously and carboplatin AUC 5 intravenously (IV) on day 1 and 22, and 5-FU 200 $\mathrm{mg} / \mathrm{m}^{2}$ continuous infusion on day $1-42$. All patients were treated prophylactic with dexamethasone $20 \mathrm{mg}$ and a 5 HT3 antagonist IV followed by ranitidine 50 $\mathrm{mg}$ IV and clemastine $2 \mathrm{mg}$ IV.

Radiotherapy was performed with a linear accelerator with a minimal photon energy of $6 \mathrm{MV}$. The total dose of $45 \mathrm{~Gy}$ was given in 25 fractions of 1.8 Gy once daily, starting on day 1 . The radiation fields encompassed the primary tumor and enlarged lymph nodes, if any, defined by endoscopy, CT scan, and 
EUS, surrounded by 5 -cm proximal and distal margins and a $2-\mathrm{cm}$ radial margin. Neoadjuvant treatment was given on a fully outpatient base.

Dose modifications were made for a toxic reaction as defined according to the criteria of the World Health Organization (WHO). ${ }^{15}$

\section{Surgery}

Surgery was planned 6-8 weeks after completion of neoadjuvant treatment. For tumors in the distal third of the esophagus, a transhiatal approach was used whenever possible. A thransthoracic Ivor-Lewis technique was used for tumors in the proximal and middle third of the esophagus. Esophagogastric continuity was reestablished using the stomach with a cervical anastomosis in all patients. A feeding jejunostomy was placed, and enteral feeding was started 24 hours after surgery.

\section{Data Collection}

The following data were prospectively collected: age, sex, WHO performance status, location of the tumor, histology of the tumor, comorbidity, toxicity of neoadjuvant treatment, type of resection, and postoperative course and complications.

\section{Histological Examination}

All pathology reports and specimens were centrally reviewed by one pathologist (GL). Pathologic assessment was performed after standard hematoxylin-eosin (H\&E) staining. The assessment included a determination of the histological type of the tumor, the depth of invasion of the tumor, whether there was nodal involvement, and the status of circumferential, proximal, and distal resection margins. The tumor stage was defined according to the classification of the American Joint Committee on Cancer. ${ }^{16}$ Patients with no residual viable tumor cells in the surgical specimen were classified as having a pathologic complete response (pCR). In addition, response after chemoradiation of the primary tumor was classified according to the Tumor Regression Grade (TRG) as described by Mandard et al. ${ }^{17}$ Categories according to the TRG ranged from TRG1, complete regression, to TRG 5 with no regressive changes. TRG 1-3 scores were defined as a major response to chemoradiation.

\section{Statistical Analysis}

The analyses were performed on the basis of intention-to-treat. Survival time was calculated as the
TABLE 1. Patient characteristics

\begin{tabular}{ll}
\hline & No. of patients $n=50$ \\
\hline Age (years) & 60 \\
Median & $34-75$ \\
Range & \\
Sex & 44 \\
Male & 6 \\
Female & 1 \\
WHO performance status & $0-2$ \\
Median & \\
Range & 24 \\
ASA classification & 24 \\
I & 2 \\
II & - \\
III & 2 \\
IV & 1 \\
Tumor location & 47 \\
Upper third & \\
Middle third & 42 \\
Lower third & 8 \\
Histology & \\
Adenocarcinoma & \\
Squamous cell carcinoma & \\
\hline
\end{tabular}

duration from the day of start of chemoradiation therapy until death or last day of follow-up, and recurrence free interval was calculated from the day of surgery until the day of diagnosis of recurrence. Overall survival and disease-free survival were estimated using the Kaplan-Meier method. Median survival time was obtained from the time corresponding to $50 \%$ survival based on the Kaplan-Meier survival curve. The log-rank test was used to assess survival differences. Significance was defined at a confidence level of $P<.05$. Statistical analyses were performed with SPSS version 15.

\section{RESULTS}

\section{Patient Characteristics}

Fifty-two patients with stage II-III esophageal carcinoma were treated in the period between January 2002 and November 2004. Two patients were found ineligible. One patient had a history of Hodgkin's disease and squamous cell carcinoma of the tongue. Previous treatment of this patient included external beam radiotherapy on the chest. One patient had intellectual disability (mental retardation) and was extremely anxious. Therefore, our study population consisted of 50 patients. All 50 patients signed the written informed consent and were included. Patient characteristics are detailed in Table 1. 
TABLE 2. Postoperative complications

\begin{tabular}{ll}
\hline Type of complication & No. of patients $(\%)$ \\
\hline Major anastomotic leakage & $5(11)$ \\
Pulmonary & $15(32)$ \\
Pneumonia & 7 \\
Emphysema & 4 \\
ARDS & 1 \\
Mediastinal infection & 1 \\
Trachea-esoph fistula & 1 \\
Chylothorax & 1 \\
Recurrent nerve palsy & $1(2)$ \\
Permanent & $4(9)$ \\
Temporary & $3(6)$ \\
Cardiac arrhythmias & $2(4)$ \\
Wound infection & $1(2)$ \\
Diaphragmatic hernia & 15 \\
Hospital stay (days) & $9-83$ \\
Median & \\
Range & 2 \\
ICU/MCU stay (days) & $2-18$ \\
Median & 1 \\
Range & $0-9$ \\
Ventilation time (days) & \\
Median &
\end{tabular}

TABLE 3. Pathological stage of patients treated with neoadjuvant chemoradiation and surgery

\begin{tabular}{lc} 
Stage, ypTNM & No. of patients $(\%)$ \\
\hline 0 & $18(38.3)$ \\
I & $6(12.8)$ \\
II a & $12(25.5)$ \\
II b & $4(8.5)$ \\
III & $7(14.9)$ \\
\hline
\end{tabular}

TABLE 4. Tumor regression grade (TRG) of patients treated with neoadjuvant chemoradiation and surgery

\begin{tabular}{lc}
\hline $\mathrm{TRG}^{a}$ & No. of patients (\%) \\
$\begin{array}{l}\text { 1-Absence of histologically identifiable } \\
\text { residual cancer and fibrosis extending }\end{array}$ & $18(38.3)$ \\
through the different layers of the \\
$\begin{array}{l}\text { esophageal wall } \\
\text { 2- Presence of rare residual cancer } \\
\text { cells scattered through the fibrosis }\end{array}$ \\
$\begin{array}{l}\text { 3- Increase in the number of residual } \\
\text { cancer cells, but fibrosis still predominated }\end{array}$ \\
$\begin{array}{l}\text { 4-Residual cancer outgrowing fibrosis } \\
\text { 5- Absence of regressive changes }\end{array}$ & $9(11(23.4)$ \\
\hline
\end{tabular}

${ }^{a}$ TRG according to Mandard et al. ${ }^{17}$

\section{Toxicity Related to Neoadjuvant Treatment}

Forty-two patients (84\%) completed full neoadjuvant treatment. Three patients discontinued chemotherapy prematurely due to grade II hand-foot syndrome, four patients due to esophagitis, and one due to grade IV leucopenia. Forty-seven patients (94\%) received at least 5 weeks chemotherapy; the remaining three patients discontinued chemotherapy in week 4 . Full dose of radiotherapy was received by 49 patients $(98 \%)$. Grade III leucopenia occurred in 23 patients (46\%), without febrile episodes. No other hematologic toxicity was recorded. All 50 patients experienced esophagitis, usually mild ( $\leq$ grade 2 ). A total of 13 patients $(26 \%)$ needed nasogastric enteral feeding. Infection of the indwelling central venous catheter occurred in two patients. There were no neoadjuvant treatment related deaths. However, one patient died of myocardial infarction 3 weeks after completing neoadjuvant treatment.

\section{Postoperative Complications}

Surgery was performed after a median of 8.1 weeks (range 6.0-17.9 weeks). No significant difference was observed between responders (TRG 1-3) and nonresponders (TRG 4-5) with respect to the time between the end of chemoradiation and surgery. Two patients showed metastatic disease at the time of surgery; hence, 47 patients underwent surgery with a curative intention. Transhiatal esophageal resection was performed in 44 patients; a transthoracic Ivor-Lewis resection was performed in three patients.

The postoperative course was uneventful in 23 patients (49\%).There were four postoperative deaths (8.5\%). Two patients died of necrosis of the gastric tube. One patient developed a fistula between the gastric conduit and the trachea and died of pulmonary sepsis. One patient died of a progressive chylothorax. Other postoperative complications included major anastomotic leakage $(n=5)$, pulmonary complications $(n=15)$, recurrent nerve palsy (temporary $n=4$, permanent $n=1$ ), and cardiac arrhythmias $(n=3)$. An overview is outlined in Table 2.

\section{Pathologic Response to Neoadjuvant Treatment}

Pathologic complete regression in the primary tumor was achieved in 18 of 47 operated patients $(38.3 \%)$. However, in two patients the primary tumor showed complete response, but microscopic residual tumor was detected in locoregional lymph nodes. The ypTNM stages of the remaining patients were: pT1N0M0 in six patients (12.8\%), pT2-3N0M0 in 12 patients $(25.5 \%)$, pT1-2N1M0 in four patients $(8.5 \%)$, and $\mathrm{pT} 3 \mathrm{~N} 1 \mathrm{M} 0 / \mathrm{pT} 4 \mathrm{~N} 0-1 \mathrm{M} 0$ in seven patients (14.9\%). Subsequent postoperative tumor stages are outlined in Table 3. Postoperative tumor regression grades (TRG) scored by an independent pathologist are outlined in Table 4. Eighteen patients (38.3\%) showed a complete tumor regression (TRG1), and 38 


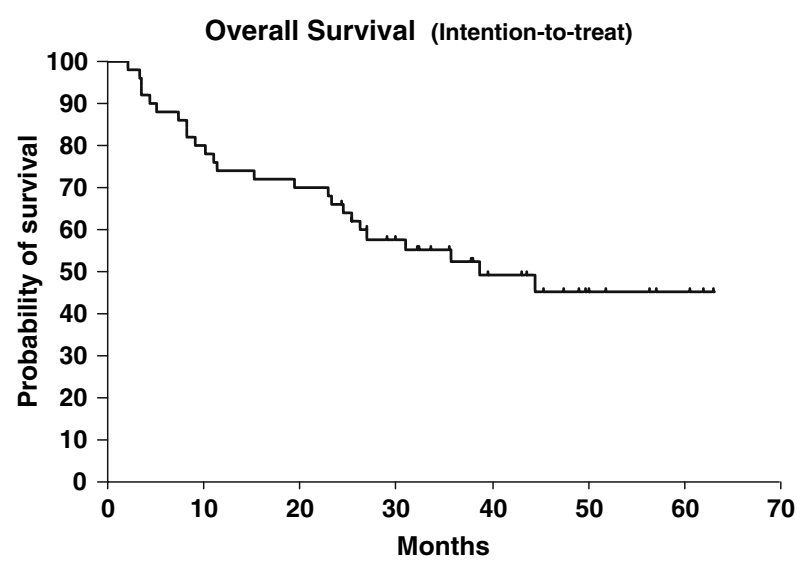

FIG. 1. Kaplan-Meier curve of overall survival of all patients $(n=50)$.

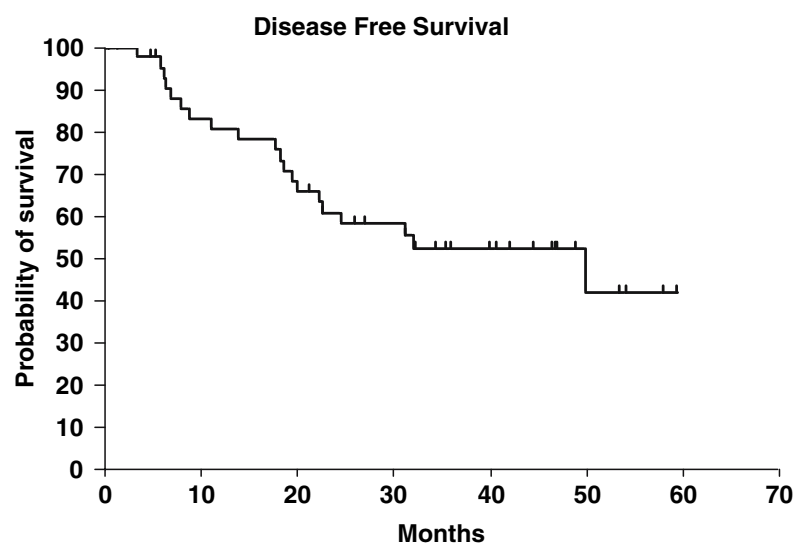

FIG. 2. Kaplan-Meier curve of disease-free survival $(n=50)$.

patients $(80.9 \%)$ displayed a major response (TRG13) after neoadjuvant treatment. Two patients had positive margin involvement of the resected specimen; therefore, a $\mathrm{R} 0$ resection was achieved in 45 of 47 patients $(95.7 \%)$.

\section{Survival and Pattern of Failure}

All 50 patients were included in the survival analysis. The median follow-up for surviving patients was 41.5 months (range 21-59 months). The median overall survival and disease-free survival were 44 and 50 months, respectively. The estimated 3- and 5-year overall survival rates were 55.6 and $47.8 \%$, respectively, whereas the estimated 3- and 5-year diseasefree survival rates were 52.2 and $41.8 \%$, respectively. The Kaplan-Meier curves for overall and disease-free survival are shown in Fig. 1 and 2.

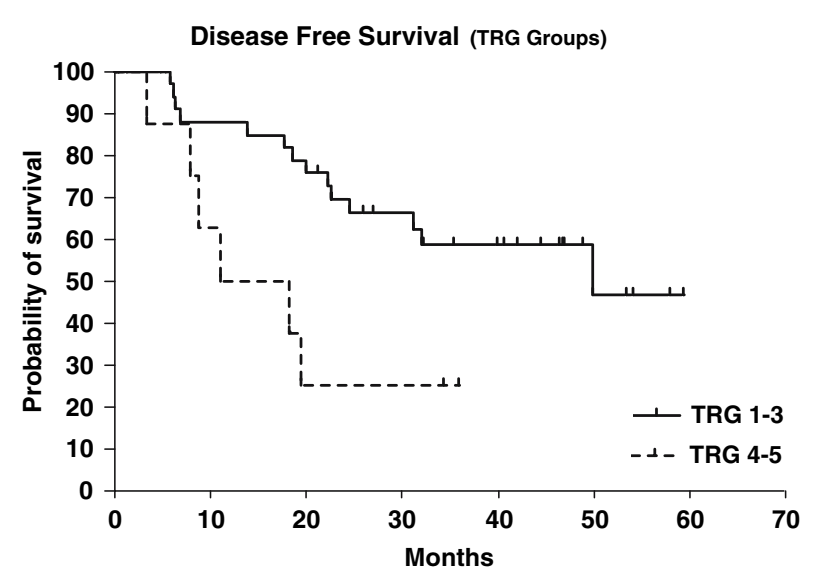

FIG. 3. Kaplan-Meier curve of disease-free survival by tumor regression grade (TRG). TRG1-3 $(n=38)$ : major response, TRG 4-5 $(n=9)$ : minor or no response $(P=.0183)$.

Patients with TRG 1-3 showed a significant improved overall and disease-free survival compared with patients with TRG 4-5. Median survival time in TRG 1-3 patients was not yet reached and 23 months in patients with TRG 4-5. The estimated 3- and 5year overall survival rates were 61.0 and $55.9 \%$ for the patients with a TRG $1-3$ and 33.3 and $16.7 \%$ for the patients with a TRG 4-5. Median disease-free survival in TRG 1-3 patients was not yet reached and 11 months in patients with TRG 4-5. The estimated 3 -year disease-free survival rates for patients with TRG $1-3$ was 58.6 vs $25.0 \%$ for patients with TRG 4 5. Comparing the TRG 1-3 patients with TRG 4-5 patients, the log-rank test of equality of the survival curves generated a $P$ value of .03 for overall survival and .02 for disease-free survival time in favor of patients with TRG1-3. The Kaplan-Meier curve for disease-free survival according to tumor regression grade is shown in Fig. 3.

Recurrent disease was found in 20 patients surviving postoperatively (20 of $43,47 \%$ ) Five of them were still alive at the time of the analysis. Recurrence was only locoregional in three patients. Distant metastases occurred in 16 patients, and in one patient recurrence presented with locoregional and pulmonary metastases.

To date 22 patients have died. Of these 22 patients, three died from nondisease-related causes and were presumed disease free. Of the 19 patients who had disease-related deaths, four patients died of postoperative complications, and three patients died of complications from local recurrence, whereas 12 patients died due to systemic failure that included metastases to lung, liver, brain, and bone. 


\section{DISCUSSION}

This study shows the results of a phase II efficacy study, with a modified chemoradiation regimen according to Meluch et al. ${ }^{6}$ A reduction of the dose of chemotherapy components of this regimen resulted in a decrease of toxicity with preservation of its high efficacy rate. The pathologic complete tumor regression rate of $38.3 \%$ and $\mathrm{R} 0$ resection rate of $95.7 \%$ are among the highest described in the literature. Toxicity and morbidity of this regimen compared with the original scheme were mild and acceptable.

Numerous phase II trials using neoadjuvant chemoradiation showed marked downstaging and suggested survival benefits. In most trials, chemotherapy regimens included cisplatin and 5-FU. However, randomized controlled trials (RCT) comparing chemoradiation followed by surgery with surgery alone have yielded inconclusive results. ${ }^{4,18-22}$ One of these trials showed significant improvement in survival in the patients who received combinedmodality therapy. ${ }^{4}$ The relevance of this study was disputed because the 3-year survival rate of $6 \%$ in the control arm was exceptionally low. Two additional trials showed a possible benefit of neoadjuvant chemoradiation without reaching a statistical significance. ${ }^{19,22}$ Furthermore, the power of these trials to detect modest but clinically relevant differences was limited by small patient numbers. In a meta-analysis report by Fiorica et al. ${ }^{23}$ six RCTs were pooled comparing neoadjuvant chemoradiation and surgery versus surgery alone. A significant difference in 3year mortality favoring neoadjuvant chemoradiation with surgery versus surgery alone was detected (OR $0.53 ; 95 \%$ CI $0.31-0.93 ; P=.03 \mathrm{NNT}=10$ ). It was concluded that neoadjuvant chemoradiation and surgery significantly improved 3-year survival compared with surgery alone in patients with resectable esophageal cancer. However, a careful interpretation of these results is warranted, because this metaanalysis was based on summarized patient data.

Neoadjuvant chemoradiation induces acute treatment-related toxicity. These effects include myelotoxicity, esophagitis, nausea, and vomiting. The regimen used in this phase II trial produced moderate toxicity and is comparable with reported literature on neoadjuvant chemoradiation regimens including paclitaxel..$^{5-7,24,25}$ The highly effective regimen used by Meluch et al. ${ }^{6}$ was modified to reduce toxicity. The modification consisted of dose reduction of 5-FU 200 $\mathrm{mg} / \mathrm{m}^{2}$ instead of $225 \mathrm{mg} / \mathrm{m}^{2}$, paclitaxel $175 \mathrm{mg} / \mathrm{m}^{2}$ instead of $200 \mathrm{mg} / \mathrm{m}^{2}$ and carboplatin AUC 5.0 instead of AUC 6.0. This modification resulted in a reduction of toxicity. Forty-two patients $(84 \%)$ received full doses of the planned 6 weeks chemotherapy, whereas in the group of Meluch et al. only 54\% of patients received full dose chemotherapy. A relative large proportion of the patients (26\%) received preoperative nasogastric enteral feeding. This was partly due to our liberal use of nasogastric enteral feeding, since in our experience optimizing preoperative nutritional status in patients treated with a neoadjuvant chemoradiaton protocol is critical. The neoadjuvant treatment was delivered on a fully outpatient basis. Further improvement of our regimen may be obtained by replacing 5-FU for novel oral fluoropyrimidines. In colorectal cancer, the novel fluoropyrimidines such as capecitabine and UFT show equal if not superior efficacy and improved patient convenience.

The reported postoperative mortality in the literature for neoadjuvant chemoradiation followed by surgery ranges from 0 to $29 \%$ (median 9\%). A nationwide study in the United States demonstrated in-hospital mortality rates of high-volume versus lowvolume centers of 8.4 vs $20.3 \% .^{2}$ A comparative Dutch study found in-hospital mortality rates of 4.9 vs $12.1 \% .{ }^{26}$ Therefore, the in-hospital mortality rate of $8.5 \%$ in our trial is within these boundaries. Several studies reported an increase in postoperative mortality after neoadjuvant chemoradiation. The aforementioned recent meta-analysis of six randomized trials confirmed a significant effect of chemoradiation on postoperative mortality (OR $2.1095 \%$ CI 1.18 $3.73, P<.01$, NNT $=25) .{ }^{23}$ A relatively short interval between completion of neoadjuvant therapy and surgery of 2-4 weeks is noted in these trials and might be of influence in the relative high mortality rates described in these trials.

We decided to perform surgery after at least 6 weeks to achieve an adequate downstaging and permit maximal recovery; in addition, surgery was postponed in some patients due to a slow recovery from the chemoradiation. We believe that the timing of surgery is important. Performing an esophagectomy too soon after neoadjuvant chemoradiation may lead to increased morbidity due to prolonged myelosuppression and hence an increased risk for developing postoperative complications.

Until recently, neoadjuvant chemoradiation studies have been performed using cisplatin/5-FU based regimens with a variation in dose and a variation in radiotherapy schemes. Moderate complete response rates of $8-26 \%$ and $\mathrm{R} 0$ resection rates of $55-84 \%$ have been described in these studies. ${ }^{4,19,20,22,27-29}$ With the implementation of regimens including pac- 
litaxel, 5-FU, and cisplatin, complete response rates of $32-38 \%$ and $\mathrm{R} 0$ resection rates of $78-95 \%$ have been documented. ${ }^{6,7,25,30}$ Our study reports a complete pathologic response of $38.3 \%$, which is high. Furthermore, a R0 resection rate of $95.7 \%$ is among the highest described in the literature. The dismal prognosis of esophageal cancer has discouraged many clinicians. For many patients, the prognosis was supposed to be dependent on the presence of indolent distant metastasis despite the type of operative treatment given. In a recent phase III trial, however, extended radical transthoracic esophagectomy showed a trend to a better survival when compared with a less radical transhiatal esophagectomy, indicating that an adequate locoregional treatment may have a beneficial effect on survival. ${ }^{3}$ However, in both groups a R0 resection rate of only $72 \%$ was noted. This observation is an important rationale for neoadjuvant combined multimodality treatment. One of the goals of neoadjuvant chemoradiation therapy is increasing curative $\mathrm{R} 0$ resection rates by downsizing the tumor and theoretically eradicating micrometastasis. Achieving $\mathrm{R} 0$ resection in locally advanced (stage II-III) esophageal cancer has been identified as a prognostic factor. ${ }^{8,9}$ Furthermore, complete pathologic response has been associated with an improved survival and local control. ${ }^{30,31}$ In the present study a major response, tumor regression grade $1-3$, resulted in a significant increase in overall and disease-free survival. Since the time between surgery and the end of the chemoradiation did not significantly differ between the responders and nonresponders, a difference in response could not be explained by a lag-time difference. Thus, a regimen with high complete pathologic response and R0-resection rates will be very likely to improve survival. However, with our relatively short followup we cannot report definitive long-term survival data yet.

In conclusion, although the discussion regarding the value of neoadjuvant therapy is ongoing, the neoadjuvant treatment regimens used for the last 20 years produced only modest benefit, at best. Improving chemoradiation regimens as in our study, by incorporating modern chemotherapeutics such as paclitaxel in combination with modern 3-D conformal radiotherapy seems to be promising. Further improvement of the systemic therapy seems to be critical as survival is mainly determined by recurrences at metastatic sites. Data of ongoing phase III trials including novel chemoradiation protocols have to be awaited in the near future.

\section{REFERENCES}

1. Reed CE. Surgical management of esophageal carcinoma. Oncologist 1999; 4:95-105.

2. Birkmeyer JD, Siewers AE, Finlayson EV, et al. Hospital volume and surgical mortality in the United States. $N$ Engl $J$ Med 2002; 346:1128-37.

3. Hulscher JB, van Sandick JW, de Boer AG, et al. Extended transthoracic resection compared with limited transhiatal resection for adenocarcinoma of the esophagus. $N$ Engl $J$ Med 2002; 347:1662-9.

4. Walsh TN, Noonan N, Hollywood D, Kelly A, Keeling N, Hennessy TP. A comparison of multimodal therapy and surgery for esophageal adenocarcinoma. $N$ Engl J Med 1996; 335:462-7.

5. Safran H, Akerman P, Cioffi W, et al. Paclitaxel and concurrent radiation therapy for locally advanced adenocarcinomas of the pancreas, stomach, and gastroesophageal junction. Semin Radiat Oncol 1999; 9:53-7.

6. Meluch AA, Greco FA, Gray JR, et al. Preoperative therapy with concurrent paclitaxel/carboplatin/infusional 5-FU and radiation therapy in locoregional esophageal cancer: final results of a Minnie Pearl Cancer Research Network phase II trial. Cancer $J$ 2003; 9:251-60.

7. Choi N, Park SD, Lynch T, et al. Twice-daily radiotherapy as concurrent boost technique during two chemotherapy cycles in neoadjuvant chemoradiotherapy for resectable esophageal carcinoma: mature results of phase II study. Int J Radiat Oncol Biol Phys 2004; 60:111-22.

8. Mulligan ED, Dunne B, Griffin M, Keeling N, Reynolds JV. Margin involvement and outcome in oesophageal carcinoma: a 10-year experience in a specialist unit. Eur J Surg Oncol 2004; 30:313-7.

9. Hofstetter W, Swisher SG, Correa AM, et al. Treatment outcomes of resected esophageal cancer. Ann Surg 2002; 236:37684.

10. Donehower RC, Rowinsky EK, Grochow LB, Longnecker SM, Ettinger DS. Phase I trial of taxol in patients with advanced cancer. Cancer Treat Rep 1987; 71:1171-7.

11. Geard CR, Jones JM, Schiff PB. Taxol and radiation. J Natl Cancer Inst Monogr 1993;89-94.

12. Choy H, Rodriguez FF, Koester S, Hilsenbeck S, Von Hoff DD. Investigation of taxol as a potential radiation sensitizer. Cancer 1993; 71:3774-8.

13. Gupta N, Hu LJ, Deen DF. Cytotoxicity and cell-cycle effects of paclitaxel when used as a single agent and in combination with ionizing radiation. Int J Radiat Oncol Biol Phys 1997; 37:885-95.

14. O'Connor PM, Jackman J, Bae I, et al. Characterization of the p53 tumor suppressor pathway in cell lines of the National Cancer Institute anticancer drug screen and correlations with the growth-inhibitory potency of 123 anticancer agents. Cancer Res 1997; 57:4285-300.

15. Miller AB, Hoogstraten B, Staquet M, Winkler A. Reporting results of cancer treatment. Cancer 1981; 47:207-14.

16. Esophagus. In Greene FL, Balch C.M., Flemming I.D., et al. (Eds). American Joint Committee on Cancer: AJCC Cancer Staging Manual. New York, NY: Springer; 2002.

17. Mandard AM, Dalibard F, Mandard JC, et al. Pathologic assessment of tumor regression after preoperative chemoradiotherapy of esophageal carcinoma. Clinicopathologic correlations. Cancer 1994; 73:2680-6.

18. Bosset JF, Gignoux M, Triboulet JP, et al. Chemoradiotherapy followed by surgery compared with surgery alone in squamouscell cancer of the esophagus. N Engl J Med 1997; 337:161-7.

19. Burmeister BH, Smithers BM, Gebski V, et al. Surgery alone versus chemoradiotherapy followed by surgery for resectable cancer of the oesophagus: a randomised controlled phase III trial. Lancet Oncol 2005; 6:659-68. 
20. Le Prise E, Etienne PL, Meunier B, et al. A randomized study of chemotherapy, radiation therapy, and surgery versus surgery for localized squamous cell carcinoma of the esophagus. Cancer 1994; 73:1779-84.

21. Nygaard K, Hagen S, Hansen HS, et al. Pre-operative radiotherapy prolongs survival in operable esophageal carcinoma: a randomized, multicenter study of pre-operative radiotherapy and chemotherapy. The second Scandinavian trial in esophageal cancer. World J Surg 1992; 16:1104-9.

22. Urba SG, Orringer MB, Turrisi A, Iannettoni M, Forastiere A, Strawderman M. Randomized trial of preoperative chemoradiation versus surgery alone in patients with locoregional esophageal carcinoma. J Clin Oncol 2001; 19:305-13.

23. Fiorica F, Di Bona D, Schepis F, et al. Preoperative chemoradiotherapy for oesophageal cancer: a systematic review and meta-analysis. Gut 2004; 53:925-30.

24. Adelstein DJ, Rice TW, Rybicki LA, et al. Does paclitaxel improve the chemoradiotherapy of locoregionally advanced esophageal cancer? A nonrandomized comparison with fluorouracil-based therapy. J Clin Oncol 2000; 18:2032-9.

25. van Meerten E, Muller K, Tilanus HW, et al. Neoadjuvant concurrent chemoradiation with weekly paclitaxel and carboplatin for patients with oesophageal cancer: a phase II study. Br J Cancer 2006; 94:1389-94.
26. van Lanschot JJ, Hulscher JB, Buskens CJ, Tilanus HW, Ten Kate FJ, Obertop H. Hospital volume and hospital mortality for esophagectomy. Cancer 2001; 91:1574-8.

27. Apinop C, Puttisak P, Preecha N. A prospective study of combined therapy in esophageal cancer. Hepatogastroenterology 1994; 41:391-3.

28. Forastiere AA, Orringer MB, Perez-Tamayo C, Urba SG, Zahurak M. Preoperative chemoradiation followed by transhiatal esophagectomy for carcinoma of the esophagus: final report. J Clin Oncol 1993; 11:1118-23.

29. Heath EI, Burtness BA, Heitmiller RF, et al. Phase II evaluation of preoperative chemoradiation and postoperative adjuvant chemotherapy for squamous cell and adenocarcinoma of the esophagus. J Clin Oncol 2000; 18:868-76.

30. Kleinberg L, Knisely JP, Heitmiller R, et al. Mature survival results with preoperative cisplatin, protracted infusion 5-fluorouracil, and 44-Gy radiotherapy for esophageal cancer. Int $J$ Radiat Oncol Biol Phys 2003; 56:328-34.

31. Berger AC, Farma J, Scott WJ, et al. Complete response to neoadjuvant chemoradiotherapy in esophageal carcinoma is associated with significantly improved survival. J Clin Oncol $2005 ; 23: 4330-7$. 